\title{
DEGENERATED LEMMA (DEL) Is a New Allele of OsRDR6 That Regulates Lemma Development and Affects Rice Grain Yield
}

Jing You

Southwest University

Qiannan Duan

Southwest University

Jun Zhang

Southwest University

Wenqiang Shen

Southwest University

Yue Zhou

Southwest University

Wenwen Xiao

Southwest University

Li Ye

Southwest University

Xinfang Zhang

Southwest University

Yan Xiang

Southwest University

Zhifeng He

Southwest University

Shunyi Song

Southwest University

Guanghua He

Southwest University

Yunfeng Li

Southwest University

Ting Zhang ( $\nabla$ tingwz@163.com )

Southwest University https://orcid.org/0000-0001-5150-3992 
Keywords: Lemma, Map-based cloning, Rice, Yield, Spikelet

Posted Date: January 7th, 2022

DOI: https://doi.org/10.21203/rs.3.rs-1147038/v1

License: (c) (i) This work is licensed under a Creative Commons Attribution 4.0 International License. Read Full License 


\section{Abstract}

The lemma and palea are floral organ structures unique to grasses, and their development affects grain size. However, information on the molecular mechanism of lemma development is limited. In this study, we investigated a rice spikelet mutant, degenerated lemma (del), which developed florets with a slightly degenerated or rod-like lemma. The results indicate that the mutation of the $D E L$ gene interfered with lemma development. In addition, del also showed a significant reduction in grain length and width, seed setting rate, and 1000-grain weight, which led to a reduction in yield. The results indicate that the mutation of the $D E L$ gene further affects rice grain yield. Map-based cloning shows a single-nucleotide substitution from $\mathrm{T}$ to $\mathrm{A}$ within Os01g0527600/DEL, causing an amino acid mutation of Leu-34 to His-34 in the del mutant. DEL is an allele of OsRDR6, encoding the RNA-dependent RNA polymerase 6, and is highly expressed in the spikelet. RT-qPCR results show that the expression of some floral organ identity genes was changed, which indicates that the $D E L$ gene regulates lemma development by modulating the expression of these genes. The present results suggest that $D E L$ plays an important role in lemma development and rice grain yield.

\section{Introduction}

Rice (Oryza sativa L.) is a model monocotyledonous plant and is one of the most important food crops in the world. The floral organs of rice are closely associated with yield and quality. An individual floret is composed of the pistil, stamen, lodicules, palea, and lemma from the innermost to the outermost whorl. The lemma and palea, also known as the glumes, are external floral organs unique to grasses and provide photosynthetic products for the floral organs in the early stages of development to protect the grain from attack by diseases and insects after maturity. In addition, the glume encloses the mature grain, and the size of the lemma and palea determines the length and width of the grain, thus the normal development of the glume is important for ensuring the normal development of the grain, and is closely related to the yield and quality (Ren et al., 2019; Shomura et al., 2008; Xing and Zhang, 2010).

The characteristic genes that regulate floral meristem identity maintain the normal development of the inflorescence, and activate the expression of the related floral organ identity genes to produce specialized floral organ primordia and ultimately form the complete floret. The so-called ABCDE model for the regulation of floral organ development in higher plants has been proposed (Theißen, 2001). Class $A$ genes are involved in the regulation of the lemma, three of which have been isolated in rice, namely OSMADS14, OSMADS15, and OSMADS18, which all belong to the SQUA gene family and the FUL subfamily. Expression of OSMADS14 can be detected in the glume and sterile lemma at the early stage of floral organ development and is expressed in the stamen and pistil at the later stage. Ectopic expression of OSMADS14 leads to an early-flowering phenotype (Jeon et al., 2000). In situ hybridization indicates that OSMADS15 is expressed in the lemma and palea; the mutant shows degeneration and albinism of the lemma, and overexpression of OsMADS15 results in early flowering (Kyozuka and Shimamoto, 2002; Wang et al., 2010). OsMADS18 is widely expressed in all tissues and its absence does not cause phenotypic abnormalities, but its overexpression leads to early flowering and accelerated tillering of the 
stem meristem (Moon et al., 1999). In addition, Class E genes are involved in the regulation of the lemma. Class E genes in rice comprise OsMADS1 (LHS1), OsMADS5, OsMADS7, OsMADS8, and OsMADS34, which predominantly regulate the development of organs in the entire floret. OsMADS1 may maintain the development of the palea and lemma and the establishment of spikelet determinacy through the production of specialized apical meristems and the differentiation and proliferation of glume cells (Malcomber and Kellogg, 2004).

The development of the lemma and palea is also regulated by the trans-acting small interfering RNA (tasiRNA) synthesis pathway. In contrast to the first five types of genes, the ta-siRNA synthesis pathway predominantly regulates lemma and palea development by affecting the establishment of polarity. The production of ta-siRNA is caused by the specific miRNA-ARGONAUTE (AGO) complex, cleaving the miRNA targets on TRANS-ACTING SIRNA transcripts (Allen et al., 2005). Subsequently, the broken single strand is synthesized into dsRNA by RDR6 under the protection of the SGS3 protein (Adenot et al., 2006; Garcia et al., 2006). Then, under the action of the DCL4 and DRB4 proteins, it is cut into siRNA to inhibit the expression of downstream target genes (Yoshikawa et al., 2005). Therefore, ta-siRNA synthesis is controlled by three types of major proteins that all play important roles, namely the AGO protein that specifically binds to miRNA; the RDR6 and SGS3 proteins involved in dsRNA formation; and the DCL4 and DRB4 proteins performing cleavage functions (Allen et al., 2005). Previous research has shown that mutations in the ta-siRNA synthesis pathway can cause defective polarity development of the glume. SHL2 encodes a protein in rice similar to Arabidopsis RDR6, which mediates the production of dsRNA. In the sh/2 mutant, the stamens and lemma are defective in adaxial-abaxial polarity development, causing the lemma to become filamentous or stick-shaped, or to fail to develop entirely (Toriba et al., 2010). Rice SHO1 and SHO2 encode proteins closely related to Arabidopsis DCL4 and AG07, respectively. Leaf growth of the sho1 mutant is accelerated and the leaves are deformed, showing short and narrow or filamentous shapes, and lemma development is hindered by the absence of adaxial surfaces (Nagasaki et al., 2007). The sho2 mutant exhibits leaf phenotypes similar to those of sho1, but lemma development has not previously been described (Song et al., 2012). Thus, genes associated with the ta-siRNA pathway are likely to be involved in the establishment of adaxial-abaxial polarity in rice.

In this study, we identified a rice DEGENERATED LEMMA (DEL) gene, a novel allele of OsRDR6, which encodes an RNA-dependent RNA polymerase, and is highly expressed in the spikelet, especially in the lemma. The del developed florets with slightly degenerated or rod-like lemma. Analysis of agronomic traits shows that grain length and width, seed setting rate, and 1000-grain weight of the de/ mutant were reduced significantly compared with the wild type. This suggests that the mutation of $D E L$ further affected rice grain yield. RT-qPCR results show that the expression of some floral organ identity genes was changed, which indicates that the $D E L$ gene regulates lemma development by modulating the expression of these genes. Taken together, these results suggest that $D E L$ regulates lemma development in rice, revealing the important role of $D E L$ in rice spikelet development and breeding.

\section{Materials And Methods}




\section{Plant materials}

The del mutant was derived from the progeny of a rice indica restorer line, Xinong 1B, treated with EMS and was stably inherited through seven successive generations of self-crossing. The $F_{1}$ progeny was generated by the cross of indica sterile line $56 \mathrm{~S}$ with del. All the plant materials used were planted in the experimental fields of the Rice Research Institute of Southwest University in Chongqing and Hainan, China and plants with mutant phenotype in $\mathrm{F}_{2}$ progeny were used to map the $D E L$ gene.

\section{Morphological and histological analysis}

At the flowering stage, living plants were collected from the field and moved to the scanning electron microscope room. Leaves wrapped in young inflorescences in living rice plants were stripped, and the spikelets were dispersed under an optical microscope. Then the spikelets were moved to the carrier table of the scanning electron microscope (Hitachi SU3500, Tokyo, Japan), and observed and photographed under low vacuum mode, $5 \mathrm{kV}$ accelerating voltage, and freezing conditions at $-20^{\circ} \mathrm{C}$.

Panicles of the wild type and the de/ mutant were collected at the heading stage and fixed in FAA solution ( $50 \%$ ethanol, $0.9 \mathrm{M}$ glacial acetic acid, and $3.7 \%$ formaldehyde) overnight at $4^{\circ} \mathrm{C}$. The fixed spikelets were dehydrated in a series of ethanol solutions, infiltrated in xylene, and embedded in paraffin (Sigma, St Louis, MO, USA). Samples were sectioned at $8 \mu \mathrm{m}$ and transferred onto poly-L-lysine-coated glass slides, deparaffinized with xylene, and dehydrated through a series of ethanol solutions. The sections were stained sequentially with $1 \%$ safranin (Amresco, Solon, OH, USA) and 1\% Fast Green (Amresco), and observed using an Eclipse E600 light microscope (Nikon).

\section{Molecular mapping of del and linkage map construction}

To localize the target gene, the bulk segregant analysis method was used (Michelmore et al., 1991). All DNA used to localize the target gene was extracted using the etyltrime thylammonium bromide method (Murray and Thompson, 1980). The InDel markers used for gene mapping were distributed equally among the 12 chromosomes. All primers were synthesized by the Beijing Tsingke Company. The total volume of PCR amplification was $13 \mu \mathrm{L}$, comprising $1.25 \mu \mathrm{L} 10 \times$ PCR buffer, $1 \mu \mathrm{L}$ of $50 \mathrm{ng} \mathrm{LL}^{-1}$ DNA, 8.5 $\mu \mathrm{LddH}_{2} \mathrm{O}, 1 \mu \mathrm{L}$ of $10 \mathrm{mmol} \mathrm{L}^{-1}$ forward and reverse primers, $0.5 \mu \mathrm{L}$ of $2.5 \mathrm{mmol} \mathrm{L}^{-1} \mathrm{dNTPs}$, and 0.1 $\mu \mathrm{L}$ of $5 \mathrm{U} \mu \mathrm{L}^{-1} \mathrm{rTaq}$ DNA polymerase. DNA amplification was achieved by the following procedure: $5 \mathrm{~min}$ at $95^{\circ} \mathrm{C}$, followed by 35 cycles of $30 \mathrm{~s}$ at $95^{\circ} \mathrm{C}, 30 \mathrm{~s}$ at $55^{\circ} \mathrm{C}$, and $1 \mathrm{~min}$ at $72^{\circ} \mathrm{C}$, with a final extension at $72^{\circ} \mathrm{C}$ for $10 \mathrm{~min}$. The PCR products were separated in $10 \%$ polyacrylamide gel and visualized after rapid silver staining (Luo et al., 2007). The relative distances between the de/ locus and the InDel markers were marked by the number of recombinants. The primers are shown in Supplemental Tables S1 and S2.

\section{Candidate genes analysis}

Information on gene annotations was obtained from the Gramene (http://www.gramene.org/) and Rice Genome Annotation Project (http://rice.plantbiology.msu.edu/) online data resources. Homology analysis 
was conducted using the BLAST tool on the National Center for Biotechnology Information website (http://blast.ncbi.nlm.nih.gov/Blast.cgi).

\section{Complementation test}

The Os01g0527600 sequence, a 7034 bp genomic fragment that contained the DEL coding sequence, coupling the $2144 \mathrm{bp}$ upstream, was downloaded from the website (http://www.gramene.org/microsat), and the forward primer DELcom-F and reverse primer DELcom-R were designed by Vector NYI Advance 10. DNA extracted from the wild type was used as template for PCR amplification and the PCR product was purified by gel extraction kit (TIANGEN BIOTECH, BEIJING). The pCAMBIA1301 original bacterial solution of the complementary vector was used for shaking replication, and the plasmid was extracted and digested by Kpnl and Xbal restriction endonuclease. Recombinant enzyme was used for ligating and transformation, and the positive strains detected by PCR were selected and sent to the Chongqing TSINGKE company for sequencing. The constructed pCAMBIA1301-DELp-DEL-GUS fusion expression vector was transformed into de/mutants by the Agrobacterium tumefaciens-mediated method. The transformation and differentiation processes were completed by the Wuhan Boyuan Company. The primer sequences used are shown in Supplemental Table S5.

\section{Quantitative real-time PCR analysis}

RNAprep Pure Plant RNA Purification Kit (Tiangen, Beijing, China) was used to isolate the total RNA of the lemma, rod-like lemma, palea, pistil, and stamen of the wild type and the del mutant. The extraction process followed the kit instructions and kept RNase free environment throughout the whole process. The purity and concentration of RNA were determined by NanoDrop ${ }^{\mathrm{TM}}$ One/One ${ }^{\mathrm{C}}$ (Thermo) and RNA integrity was determined by agarose gel electrophoresis. 2 ug of purified RNA was reverse-transcribed into cDNA using the PrimeScript ${ }^{\circledR}$ Reagent Kit with gDNA Eraser (TaKaRa). Half a microliter of the reversetranscribed RNA was used as a PCR template with gene-specific primers (Table S4). Quantitative real-time PCR analysis was performed in three replicates using the SYBR® Premix Ex Taq ${ }^{\text {TM }}$ II Kit (TaKaRa, Dalian, China) with the CFX Connect ${ }^{\text {TM }}$ Real-Time System (Bio-Rad). ACTIN was used as an internal reference gene (Table S4). The complete reaction conditions followed the kit instructions. The average expression level was calculated for each gene. All samples were obtained from fresh plants in the field, stored in liquid nitrogen, and transported to the laboratory. RNA extraction, reverse transcription and RT-qRCR experiments were completed within two days. Residual RNA samples were stored at $-80^{\circ} \mathrm{C}$ and $\mathrm{cDNA}$ at $-20^{\circ} \mathrm{C}$.

\section{In situ hybridization}

Young panicles from the wild type were fixed in 70\%FAA (RNase free), then dehydrated through a series of alcohols/xylenes, before being embedded in paraffin (Sigma-Aldrich). For the DEL probe, gene-specific cDNA was amplified and labeled using the DIG RNA Labeling Kit (Roche, Basel, Switzerland). The in situ hybridization was performed as described previously (Zhang et al., 2017). The primers are shown in Supplemental Table S5. 


\section{Results}

\section{Morphological and histological analysis of the del mutant at the flowering stage}

The spikelet of the wild type contains one terminal fertile floret, one pair of sterile lemmas, and one pair of rudimentary glumes in rice (Fig. 1-A). The terminal fertile floret is bisexual and consists of one pistil and six stamens, as well as two lodicules and one pair of bract-like organs (lemma and palea) (Fig. 1-B and C). There are five and three vascular bundles in the lemma and palea, respectively. In addition, the lemma is composed of four cell layers: from the abaxial to the adaxial surface these are a silicified upper epidermis, sclerenchyma cells, parenchyma cells, and a vacuolated inner epidermis, and the palea is composed of two fused parts: the body of the palea (bop) and two marginal regions of the palea (mrp) (Fig. 1-D and E). The cellular structure of the bop is similar to that of the lemma, but the mrp displays a distinctive smooth epidermis. The lemma and palea are hooked together to protect the inner floral organs and grain (Fig. 1-F and G).

In comparison with the widle type, in the de/ mutant $36 \%$ of spikelets developed a slightly degenerated lemma (Fig.1-H and N), which was narrow and unable to hook with the palea, causing a cracked floret (Fig.1-H and J). The palea and inner floral organs developed normally (Fig.1-I, K and L). Inspection of paraffin sections revealed that the four-layer cell structure of the lemma persisted, but the number and volume of the cells were significantly lower in the mutant (Fig. 1-M and N). In 53\% of spikelets, a rod-like lemma formed (Fig.1-O and T), and in these spikelets, the lemma was extremely degenerated and transformed into an awn-like organ (Fig.1-0, Q and R). The morphology of the lemma was highly similar to that of the awn and contained only one midvein. The silicified cells that should have been present on the outside surface of the wild type were ectopic and found in the inner epidermis of the rod-like lemma. The internal cell structure was almost completely disordered and unrecognizable (Fig.1-T and U). The palea and inner floral organs in the mutant were normal (Fig.1-P, S and T), thus the del mutant showed degeneration of the lemma to differing degrees, which suggests that $D E L$ plays an important role in lemma development.

\section{The del mutant affects grain yield}

The spike type of the de/mutant was more erect and smaller than that of the wild type. Compared with the wild type, the plant height of the de/ mutant was increased by $6.55 \mathrm{~cm}$, while the panicle length was reduced by $24.5 \%$ (Fig. 2-A, B, E and F). While the number of primary branches of del showed no significant difference, the number of secondary branches was reduced by $10.7 \%$ (Fig. $2-G$ and $H$ ). The rate of seed setting in de/ was reduced by $70 \%$ (Fig. 2-I). As well as impaired flower development, the mature grains of $d e /$ were also affected.The glumes of the mature grains in de/ were still dehiscent, and the lemma was rod-like in the severe mutant (Fig. 2-C). The mature brown rice grains were shaped like water droplets, being narrow at the top and wide at the bottom, and the rod-like spikelets produced few grains (Fig. 2-D). The grain length and the brown-rice grain length of the de/ were reduced by $7.7 \%$ and $27.0 \%$, respectively (Fig. 2-J and M). The grain width of the de/ was increased by $16.3 \%$, but the brownrice grain width of the de/ was reduced by $26.8 \%$ (Fig. 2-K and N). The 1000-grain weight and the 1000- 
grain brown-rice weight were also reduced by $71.3 \%$ and $83.0 \%$, respectively (Fig. 2 -L and 0 ). The results reveal that the $D E L$ gene plays an important role in spikelet development and affects rice grain yield.

\section{Early morphological analysis of the del mutant}

Using scanning electron microscopy, we examined spikelets of the wild type and de/mutant during the early developmental stages. In the wild-type floret, the primordia of the lemma and palea began to develop during the spikelet 4 (Sp4) stage (Fig.3-A). At Sp4, no significant difference between spikelets of the wild type and the de/ mutant was observed (Fig.3-A, E, and I). In the wild-type floret, the lemma and palea primordia were formed and the palea was smaller than the lemma, the five spherical stamen primordia were formed synchronously during the Sp5 and Sp6 stages except for the primordium nearest the lemma, and the lodicule primordia were formed at a subsequent stage (Fig.3-B). In the de/ mutant, which was different from the wild type, the shape of the slightly degenerated lemma was normal (Fig.3F), and the margin of the rod-like lemma was not developed and did not intersect with the palea primordium (Fig.3-J). During the Sp7 stage, the pistil primordium was initiated and the lemma and palea primordia were normally developed and semi-closed, enclosing the inner whorl organ primordia in the wild-type floret (Fig.3-C). In the de/ mutant, the slightly degenerated lemma primordium was noticeably shorter and narrower than the lemma primordium in the wild type (Fig.3-G), cell differentiation on each side of the rod-like lemma was slow, and development into a rod-like structure occurred gradually (Fig.3$\mathrm{K})$. During the Sp8 stage, the single floret of the wild-type spikelet underwent a further stage of development (Fig.3-D). In the de/mutant, the slightly degenerated lemma was significantly narrowed, and the palea and lemma were unable to close to cover the inner floral organs (Fig.3-H). The rod-like lemma primordium was further elongated, but the flanks remained undeveloped, and the inner floral organs were completely bare. However, the inner floral organs were normal (Fig.3-L). Collectively, these observations reveal that the defects of the lemma in the de/ mutant arose during the early stages of spikelet development.

\section{Map-based cloning of DEL}

The $\mathrm{F}_{1}$ progeny was derived from the cross between $56 \mathrm{~S}$ and the de/ mutant, and showed a wild-type phenotype. The segregation ratio of the normal to the mutant phenotype was 3:1 (1091 wild-type-like plants and 358 mutant-like plants; $\chi^{2}=0.26<\chi_{0.05,1}^{2}=3.84$ ) in the $F_{2}$ population. This result indicates that the del mutant phenotype was controlled by a single recessive gene.

A map-based cloning approach was used to fine-map the $D E L$ gene. The 358 recessive individuals in the $\mathrm{F}_{2}$ population were used as a mapping population to localize the DEL gene. To screen for polymorphism between the parents, a total of 420 pairs of simple sequence repeat (SSR) and InDel primers evenly distributed in the rice genome were used (Table S1). Ninety InDel markers were selected and used to further screen two DNA pools prepared by mixing equal amounts of genomic DNA from either 10 wildtype-like $F_{2}$ plants or 10 mutant-like $F_{2}$ plants (Table S2). The $D E L$ gene was localized between the InDel markers In1-18.68 and In1-20.376 on chromosome 1 (Fig. 4-A and B). To fine-map DEL, 30 pairs of InDel 
primers located between $\ln 1-18.68$ and $\ln 1-20.376$ were developed, of which $\ln 1-18.68, \ln 1-18.79$, In118.94, In1-19.08, and In1-20.38 exhibited polymorphism between the parents (Table S3). Among all the $F_{2}$ individuals, these five markers detected separately 14, 8, 4, 12, and 23 recombinants (Fig. 4-C). These results show that $D E L$ was located between the InDel markers of $\ln 1-18.94$ and $\ln 1-19.08$. The estimated physical distance between In1-18.94 and In1-19.08 was approximately $140 \mathrm{~kb}$, and 15 annotated genes (RAPdb annotation) were included within this interval: four enzymes (Os01g0527600, 0s01g0527700, Os01g0528800, Os01g0529800), five expressed protein (Os01g0528300, Os01g0528700, Os01g0529700, Os01g0530100, Os01g0530200), a transcription factor (Os01g0528000), two transposon protein (Os01g0527900, Os01g0529400), a non-protein coding transcript (Os01g0527801), a hypothetical protein (Os01g0530000), and a gene with no annotated information (Os01g0529101) (Fig. 4-D and Table S6).

Sequencing analysis showed that 0 s01g0527600 had a single-nucleotide substitution from $\mathrm{T}$ to $\mathrm{A}$, which was an allele of OsRDR6. The substitution of the nucleotide substitution in the del mutant caused the amino acid mutation of Leu-34 to His-34.In order to confirm that the mutation of Os01g0527600caused the de/ mutant phenotypes, we cloned a fragment consisting of a 2144-bp upstream sequence from the start codon and 4890-bp coding region sequence of the 0 s01g0527600gene in the wild type into the pCAMBIA1301 vector with the green fluorescent protein (GUS). The recombinant plasmid was then introduced into the delmutant (Fig.4-F). Subsequently, a total of 13 complementary transgenic lines were obtained, and GUS staining showed that 7 lines of them appeared indigo blue. This indicated that these lines had correctly transformed and successfully transferred into exogenous target vectors. All the $T_{0}$ positive plants were planted in the fields, and the spikelet phenotype of these 7 lines was restored to the wild-type phenotype (Fig.4-G). These results confirm that the 0 s01g0527600 gene is the DEL gene, and the phenotype of $d e /$ is indeed caused by mutation of the Os01g0527600 gene.

\section{Spatiotemporal expression pattern of DEL}

In order to clarify the functions of the DEL gene, we used RT-qPCR and in situ hybridization to test for DEL expression in the wild type. RT-qPCR analysis showed that DEL was constitutively expressed in whole organs, including root, stem, blade, sheath, and panicles. The lowest expression was found in the stem, and the highest expression was found in the blade. The expression level of the sheath was slightly higher than that of the stem, and the expression levels of the root and panicle were similar (Fig.5-A). In the spikelet, DEL was expressed in the lemma, palea, pistil, stamen, and lodicule (Fig.5-B). Later, in situ hybridization was used to detect the expression pattern of $D E L$, and a strong signal was shown in the rice spikelet (Fig.5-C-F). At the Sp3 stage, the DEL gene was highly expressed in the rudimentary glume, the sterile lemma, and the floral meristem (Fig.5-C). At the Sp4 stage, a strong DEL signal was detected in the lemma and palea primordia and floral meristem (Fig.5-D). At Sp5-Sp8, DEL was expressed in the lemma, palea, stamen, pistil, and lodicule (Fig.5-E-F). These results indicate that $D E L$ was mainly expressed in the spikelet and florets, and was highly expressed throughout the period of plant growth, which implies its role in regulating the development of the lemma. Because del showed a phenotype of lemma degeneration to differing degrees, we also explored the expression of $D E L$ in the wild-type glume. The 
results show that $D E L$ was highly expressed in the whole of the lemma and palea, especially in their eight vascular bundles (Fig. 5-F).

\section{Expression analysis of floral organ identity genes in the del mutant and wild type}

Given that the de/ mutant showed an abnormal phenotype of floral organs, the expression patterns of known genes associated with floral organ identity were measured. $D L$ (which is expressed in the middle of lemma)expression was $150 \%$ higher in the slightly degenerated lemma of the de/ mutant than in the lemma of the wild type, but the expression level of $D L$ in the rod-like lemma was greatly reduced, with only a basal level similar to that of the wild-type palea (Fig.6-A). Moreover, the expression level of OsMADS1 in both the slightly degenerated and the rod-like lemma was higher than that of the wild-type lemma, by a factor of 2 and 12, respectively. The expression level of OSMADS1 in the del palea was only twice as high as that of the wild type (Fig.6-B). OsMADS14 expression was similar to OsMADS1, and the expression of the slightly degenerated and the rod-like lemma was much higher than that of the wild-type lemma. There was no significant difference between the expression of the palea in the wild type and del (Fig.6-C). For the expression level of OSMADS15, there was little difference between the rod-like and the wild-type lemma, but the expression level of the slightly degenerated lemma was 2.5 times that of the wild-type lemma, and the expression level of the palea in de/was 1.5 times that of the wild-type palea (Fig.6-D). There was no difference in expression between the two types of de/lemma and the wild-type lemma, and the expression of the palea in the de/ palea was about $70 \%$ higher than that of the wild-type palea (Fig.6$\mathrm{E})$. These results suggest that the slightly degenerated lemma retained the identity of the lemma, and degeneration of the rod-like lemma in the de/ mutant may be caused by reduced expressionof

DL.OSMADS6 (which is an mrp identity gene) was expressed in the palea of the wild type and de/mutant, which indicates that the palea identity in the del mutant was normal (Fig.6-E). In addition, we also assessed the expression of OsMADS2, OsMADS3, and OsMADS4 (the stamen and pistil genes). Compared with the wild type, the expression of OSMADS2 and OSMADS3 was reduced by a factor of 2 and 4 in the pistil of del, and was increased by a factor of 111 and 59 in the stamens of del (Fig.6-F and G). The expression level of OsMADS4 was slightly higher in the de/ than in the wild-type pistil, but was 50 times higher in the de/ than in the wild-type stamen, which indicates that the stamen and pistil of the del mutant showed normal identities (Fig.6-H). Collectively, these results indicate that the de/ mutation plays a critical role in the regulation of $D L$ for lemma identity.

\section{Discussion}

In our study, we identified a mutant del, which contains a mutant allele of OsRDR6, in order to gain a better understanding of the functions of OsRDR6. The del mutant had the same phenotypes as the reported osrdr6 mutants, such as cracked glumes, caused by the different degrees of degradation of the lemma (needle/rod/awe-like structure). Moreover, the de/ mutant also exhibited an altered grain size. We suggest that the different backgrounds and mutation sites of the de/ mutant and the osrdr6 mutants could result in additional phenotypes. SHL2 encodes OsRDR6, eight sh/2 alleles (sh/2-1 through sh/2-8) from 0 . sativa cultivar Taichung 65 have been identified, and most alleles have a nonsense mutation, 
frameshift mutation, or amino acid substitution in the conserved RdRP domain. The sh/2 mutants reported to date are embryonic or seedling-lethal, caused by a failure in the formation of the SAM in the embryo. For the osrdr6-1 mutant from japonica cultivar Zhonghua11, a single nucleotide transition from $\mathrm{G}$ to $\mathrm{T}$ in osrdr6-1 leads to substitution of a highly conserved tryptophan to cysteine. Most spikelets in osrdr6-1 showed needle-shaped, awn-like lemma and altered stamen number. The sh/2-ro/ mutant from the japonica cultivar Nipponbare has an amino acid substitution in the $\mathrm{N}$-terminal region far from the RdRP domain. In the shl2-rol spikelets, morphological defects were observed in the lemma (needle-like structure), palea, and stamen, resulting in a lack of organs or a reduction in the number of them. By comparison, we considered the del from the indica restorer line, Xinong 1B (a local variety of Chongqing). Substitution of the nucleotide in the de/ mutant caused amino acid mutation of Leu-34 to His-34 in the Nterminal region far from the RdRP domain. The de/ mutant displayed degenerated lemma and abnormal grains, whereas the palea and all the inner whorl organs (stamen, pistil, lodicule) developed in normal shapes and numbers. This study provides some new insights into the role of OsRDR6, allowing a better understanding of the development and formation of the lemma and grain.

Improved grain yield is an important goal for basic and applied scientific research in plants (Ren et al., 2019). Although several genes that affect grain size in rice have been identified, the molecular mechanisms remain unclear. Thus, it is still difficult to improve grain yield using these reported genes in rice. Normal development of floral organs, specifically the glumes (i.e., lemma and palea) is the basis for ensuring the normal development of the grain, and is also closely related to the yield (Ren et al., 2019; Shomura et al., 2008; Xing and Zhang, 2010). Grain size is also an important factor in determining rice yield. In our study, compared with the wild type, the grain length and the brown-rice grain length of the del were reduced by $7.7 \%$ and $27.0 \%$, respectively. The grain width of the de/ was increased by $16.3 \%$, but the brown-rice grain width of the de/ was reduced by $26.8 \%$. The 1000 -grain weight and the 1000 -grain brown rice weight were also reduced by $71.3 \%$ and $83.0 \%$, respectively. The reduced grain or brown-rice grain length and width in del may be due to the degenerated lemma, which provides photosynthetic products for the floral organs in the early stages of development, to protect the grain from attack by diseases and insects after maturity, and determines the length and width of the grain. Taken together, the normal function of the $D E L$ gene is the basis for the normal development of lemma morphology and size, and has an important influence on rice grain yield. $D E L$ is thus a possible target for efforts to improve rice grain yield.

The lemma and palea are specialized floral organs that are unique to grasses. The development of the lemma has been an important focus of research on floral organ development in rice. In previous research, LHS1 (OsMADS1) was shown to regulate the formation and development of the lemma, predominantly by controlling the differentiation of specific cells of the lemma (Jeon et al., 2000). The complete loss-offunction of OSMADS1 results in a homologous transformation of the three internal whorls of floral organs (lodicules, stamens, and pistil) into a lemma and palea-like structure (Agrawal et al., 2005; Jeon et al., 2000). The $D L$ gene is a member of the YABBY family. The phenotype of the $d l$-sup 6 mutant differs from that of other mutants in that a DNA fragment is inserted into the second intron of $D L$, resulting in loss of expression of $D L$ and a change in the number of vascular bundles of the lemma (Ohmori et al., 2011). 
Compared with the aforementioned mutants, the de/ mutant displays a lemma degenerated to different degrees, including a rod-like lemma, and the surface of the lemma is similar to that of the awn. However, the palea and inner floral organs are normally developed. DEL is the allele of OSRDR6, which encodes an RNA-dependent RNA polymerase, and is essential for ta-siRNA synthesis in rice. Genes associated with the ta-siRNA pathway are likely to be involved in the establishment of adaxial-abaxial polarity. Rice SHO1 and SHO2 encode proteins closely related to Arabidopsis DCL4 and AG07, respectively. Leaf growth of the sho1 mutant is accelerated and the leaves are deformed, showing short and narrow or filamentous shapes, and lemma development is hindered by the absence of adaxial surfaces (Nagasaki et al., 2007). The sho2 mutant exhibits leaf phenotypes similar to those of sho1 (Song et al., 2012). OsDCL 4 is responsible for the formation of the 21-nucleotide-long siRNA. In the osdcl4-1 mutant, the lemma shows degeneration and widespread conversion to an awn (Liu et al., 2007). Similar to the above mutations in ta-siRNA synthesis pathway, the lemma of the del mutant was also degenerated to varying degrees, being partially or completely degenerated to the awn. Therefore, the function of the ta-siRNA synthesis pathway is conserved, in regulating lemma and palea development by affecting the establishment of polarity.

\section{Conclusion}

In this study, we identified a mutant, named del, related to the spikelet development with degenerated lemma. In addition, del also showed a significant reduction in grain length and width, seed setting rate, and 1000-grain weight, which led to a reduction in yield. Mapping, cloning, and sequencing revealed that $D E L$ is an allele of OSRDR6, encoding the RNA-dependent RNA polymerase 6, and is highly expressed in the spikelet. The expression analysis indicated that the $D E L$ gene regulates lemma development by modulating the expression of some floral organ identity genes. The present results suggest that $D E L$ plays an important role in lemma development and rice grain yield.

\section{Declarations}

\section{Funding}

This work was supported by the National Natural Science Foundation of China $(31900612,31730063$, and 31971919), and Natural Science Foundation of Chongqing, China (cstc2021jcyj-bsh0147). The authors would like to thank reviewers of the manuscript who helped improve the presentation.

\section{Conflict of interest}

The authors declare that they have no conflict of interest.

\section{Author contributions}

All authors contributed to the study conception and design. Jing You and Ting Zhang made substantial contributions to the conception or design of the work. Material preparation was performed by Yue Zhou, 
Wenwen Xiao and Li Ye. Phenotypic experiment was performed by Qiannan Duan, Xinfang Zhang and Jun Zhang. Wenqiang Shen and Zhifeng He made the creation of new software used in the work. Data collection and analysis were performed by Jing You, Yan Xiang and Shunyi Song. The first draft of the manuscript was written by Jing You and Ting Zhang and all authors commented on previous versions of the manuscript. All authors read and approved the final manuscript. Guanghua He and Yunfeng Li agreed to be accountable for all aspects of the work in ensuring that questions related to the accuracy or integrity of any part of the work are appropriately investigated and resolved.

\section{Compliance with ethical standards}

We declare that the experiments obey the ethical standards of the Rice Research Institute, Key Laboratory of Application and Safety Control of Genetically Modified Crops, Academy of Agricultural Sciences, Southwest University.

\section{Acknowledgments}

This work was supported by the National Natural Science Foundation of China (31900612, 31730063, and 31971919), and Natural Science Foundation of Chongqing, China (cstc2021jcyj-bsh0147).

\section{References}

1. Adenot X, Elmayan T, Lauressergues D, Boutet S, Bouché N, Gasciolli V and Vaucheret $H$ (2006) DRB4-Dependent TAS3 trans-Acting siRNAs Control Leaf Morphology through AG07. Current Biology 16:927-932.

2. Agrawal GK, Abe K, Yamazaki M, Miyao A and Hirochika H (2005) Conservation of the E-function for Floral Organ Identity in Rice Revealed by the Analysis of Tissue Culture-induced Loss-of-Function Mutants of the OsMADS1 Gene. Plant Molecular Biology 59:125-135.

3. Allen E, Xie Z, Gustafson A and Carrington J (2005) microRNA-Directed Phasing during Trans-Acting siRNA Biogenesis in Plants. Cell121:207-221.

4. Garcia D, Collier SA, Byrne ME and Martienssen RA (2006) Specification of Leaf Polarity in Arabidopsis via the trans-Acting siRNA Pathway. Current Biology 16:933-938.

5. Jeon J-S, Jang S, Nam J, Kim C, Lee S-K, Chung Y-Y, Kim R, Lee Y, Cho Y-G and An G (2000) leafy hull sterile 1 Is a Homeotic Mutation in a Rice MADS Box Gene Affecting Rice Flower Development. The Plant cell 12:871-884.

6. Jiang L, Qian D, Zheng H, Meng LY, Chen J, Le WJ, Zhou T, Zhou YJ, Wei CH and Li Y (2012) RNAdependent RNA polymerase 6 of rice (Oryza sativa) plays role in host defense against negativestrand RNA virus, Rice stripe virus. Virus research 163:512-519.

7. Kyozuka J and Shimamoto K (2002) Ectopic Expression of OsMADS3, a Rice Ortholog of AGAMOUS, Caused a Homeotic Transformation of Lodicules to Stamens in Transgenic Rice Plants. Plant and Cell Physiology 43:130-135. 
8. Li H, Liang W, Jia R, Yin C, Zong J, Kong H and Zhang D (2010) The AGL6-like gene OsMADS6 regulates floral organ and meristem identities in rice. Cell Research 20:299-313.

9. Liu B, Chen Z, Song X, Liu C, Cui X, Zhao X, Fang J, Xu W, Zhang H, Wang X, Chu C, Deng X, Xue Y and Cao X (2007) Oryza sativa dicer-like4 reveals a key role for small interfering RNA silencing in plant development. Plant Cell 19:2705-2718.

10. Luo ZK, Yang ZL, Zhong BQ, Li YF, Xie R, Zhao FM, Ling YH and He GH (2007) Genetic analysis and fine mapping of a dynamic rolled leaf gene, RL10(t), in rice (Oryza sativa L.). Genome 50:811-817.

11. Malcomber S and Kellogg E (2004) Heterogeneous expression patterns and separate roles of the SEPALLATA gene LEAFY HULL STERILE1 in grasses. The Plant cell 16:1692-1706.

12. Michelmore RW, Paran I and Kesseli RV (1991) Identification of Markers Linked to DiseaseResistance Genes by Bulked Segregant Analysis - a Rapid Method to Detect Markers in Specific Genomic Regions by Using Segregating Populations. Proceedings of the National Academy of Sciences of the United States of America 88:9828-9832.

13. Moon YH, Kang HG, Jung JY, Jeon JS, Sung SK and An G (1999) Determination of the motif responsible for interaction between the rice APETALA1/AGAMOUS-LIKE9 family proteins using a yeast two-hybrid system. Plant physiology 120:1193-1204.

14. Murray MG and Thompson WF (1980) Rapid Isolation of High Molecular-Weight Plant DNA. Nucleic acids research 8:4321-4325.

15. Nagasaki H, Itoh J, Hayashi K, Hibara K, Satoh-Nagasawa N, Nosaka M, Mukouhata M, Ashikari M, Kitano H, Matsuoka M, Nagato $Y$ and Sato $Y(2007)$ The small interfering RNA production pathway is required for shoot meristem initiation in rice. Proc Natl Acad Sci U S A 104:14867-14871.

16. Ohmori Y, Toriba T, Nakamura H, Ichikawa H and Hirano HY (2011) Temporal and spatial regulation of DROOPING LEAF gene expression that promotes midrib formation in rice. Plant $J$ 65:77-86.

17. Prasad K, Parameswaran S and Vijayraghavan U (2005) OsMADS1, a rice MADS-box factor, controls differentiation of specific cell types in the lemma and palea and is an early-acting regulator of inner floral organs. Plant J 43:915-928.

18. Satoh N, Itoh J and Nagato Y (2003) The SHOOTLESS2 and SHOOTLESS1 genes are involved in both initiation and maintenance of the shoot apical meristem through regulating the number of indeterminate cells. Genetics 164:335-346.

19. Song X, Wang D, Ma L, Chen Z, Li P, Cui X, Liu C, Cao S, Chu C, Tao Y and Cao X (2012) Rice RNAdependent RNA polymerase 6 acts in small RNA biogenesis and spikelet development. Plant $J$ 71:378-389.

20. Theißen G (2001) Development of floral organ identity: stories from the MADS house. Current Opinion in Plant Biology 4:75-85.

21. Toriba T, Suzaki T, Yamaguchi T, Ohmori Y, Tsukaya H and Hirano H-Y (2010) Distinct Regulation of Adaxial-Abaxial Polarity in Anther Patterning in Rice. The Plant Cell 22:1452-1462.

22. Wang K, Tang D, Hong L, Xu W, Huang J, Li M, Gu M, Xue Y and Cheng Z (2010) DEP and AFO regulate reproductive habit in rice. PLoS genetics 6:e1000818. 
23. Yoshikawa M, Peragine A, Park MY and Poethig RS (2005) A pathway for the biogenesis of transacting siRNAs in Arabidopsis. Genes \& development 19:2164-2175.

24. Jeon JS, Jang S, Lee S, Nam J, Kim C, Lee SH, Chung YY, Kim SR, Lee YH, Cho YG and An G (2000) leafy hull sterile1 is a homeotic mutation in a rice MADS box gene affecting rice flower development. Plant Cell 12:871-884.

25. Ren D, Cui Y, Hu H, Xu Q, Rao Y, Yu X, Zhang Y, Wang Y, Peng Y, Zeng D, Hu J, Zhang G, Gao Z, Zhu L, Chen G, shen L, Zhang Q, Guo L and Qian Q (2019) AH2 encodes a MYB domain protein that determines hull fate and affects grain yield and quality in rice. The Plant Journal 100:813-824.

26. Shomura A, Izawa T, Ebana K, Ebitani T, Kanegae H, Konishi S and Yano M (2008) Deletion in a gene associated with grain size increased yields during rice domestication. Nature genetics 40:1023-1028.

27. Xing Y and Zhang Q (2010) Genetic and molecular bases of rice yield. Annual review of plant biology 61:421-442.

28. Zhang T, Li Y, Ma L, Sang X, Ling Y, Wang Y, Yu P, Zhuang H, Huang J, Wang N, Zhao F, Zhang C, Yang $Z$, Fang L and He G (2017) LATERAL FLORET 1 induced the three-florets spikelet in rice. Proceedings of the National Academy of Sciences 114:201700504.

\section{Figures}



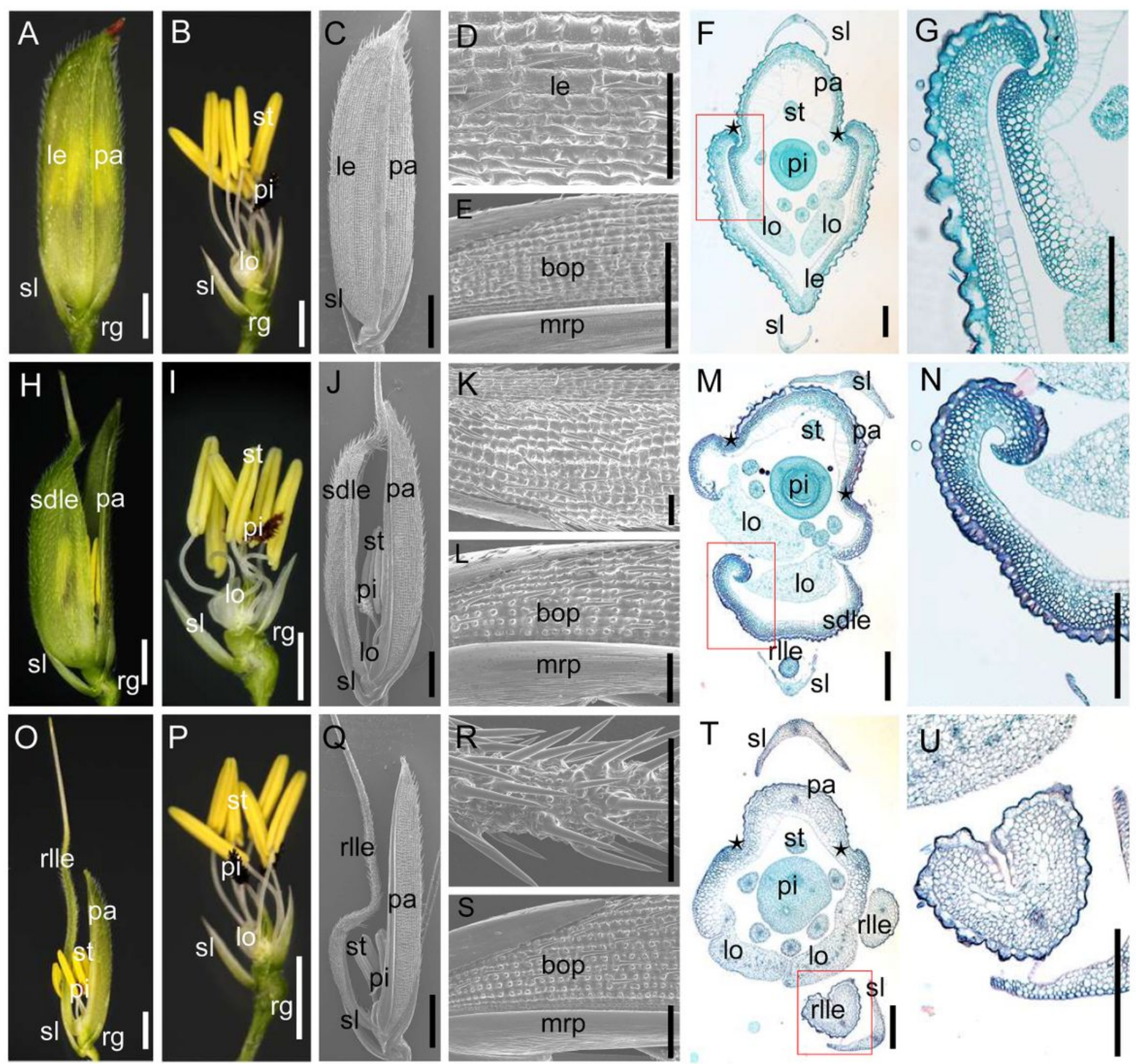

\section{Figure 1}

\section{Figure 1}

Phenotype of spikelets of the wild type and the de/ mutant. A, Complete spikelet of the wild type. B, The spikelet shown in A with the lemma and palea removed. C, Surface of wild-type spikelet. D, Surface features of the lemma. E, Surface features of the palea. F, Transverse sections of a wild-type spikelet. G, High-magnification image of the area in the red box in F. H, A del mutant spikelet with a slightly degenerated lemma. I, The spikelet shown in $\mathrm{H}$ with the lemma and palea removed. J, Surface of the slightly degenerated lemma with the inner floral organs exposed. K, Surface features of the slightly 
degenerated lemma. L, Surface features of the palea. $M$, Transverse sections of a de/ mutant spikelet. N, High-magnification image of the area in the red box in M. O, A del mutant spikelet with a rod-like lemma. $P$, The spikelet shown in $O$ with the lemma and palea removed. $Q$, The surface of the rod-like lemma with the inner floral organs exposed. R, Surface features of the rod-like lemma. S, Surface features of the palea. T, Transverse sections of a de/ mutant spikelet. $U$, High-magnification image of the area in the red box in T. Black stars indicate the joining of the lemma and palea. le, lemma; pa, palea; sl, sterile lemma; rg, rudimentary glume; st, stamen; pi, pistil; lo, lodicule; sdle: slightly degenerated lemma organ; rlle: rodlike lemma organ. Bars $=1 \mathrm{~cm}$ in $\mathrm{A}-\mathrm{C}, \mathrm{H}-\mathrm{J}, \mathrm{O}-\mathrm{Q} ; 2 \mathrm{~mm}$ in $\mathrm{D}, \mathrm{E}, \mathrm{G}, \mathrm{K}, \mathrm{L}, \mathrm{N}, \mathrm{R}, \mathrm{S}$ and $\mathrm{U} ; 500 \mu \mathrm{m}$ in $\mathrm{F}, \mathrm{M}$, and $\mathrm{T}$.
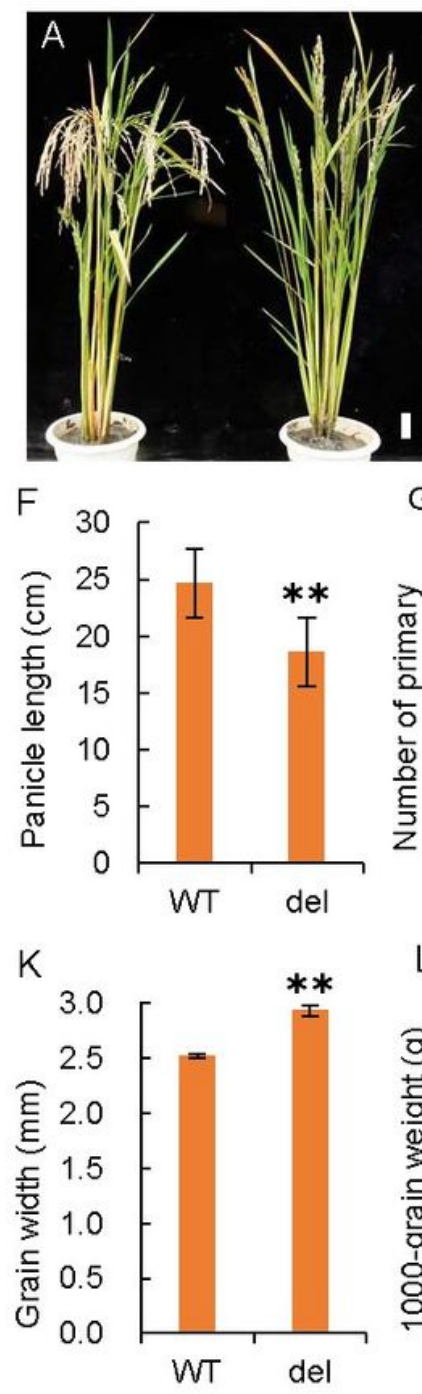
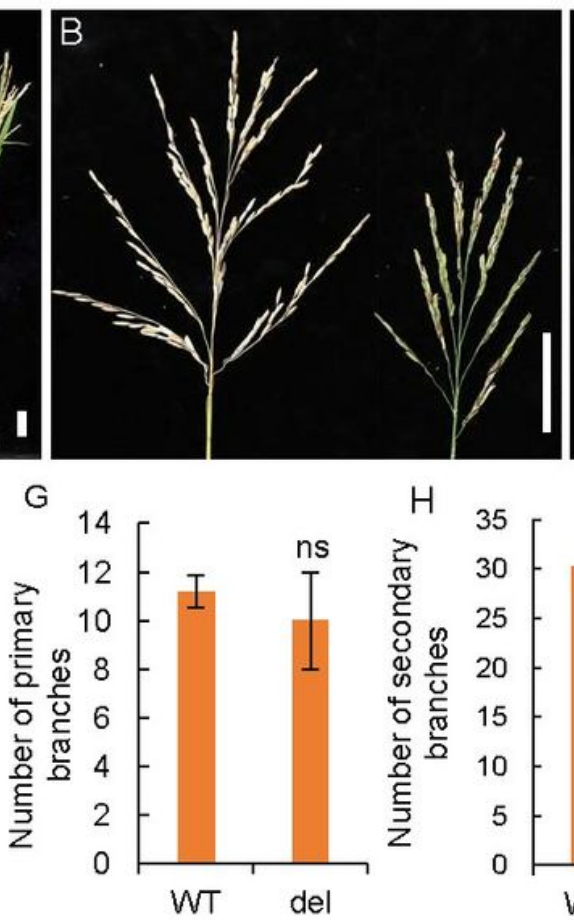

$\mathrm{L}$

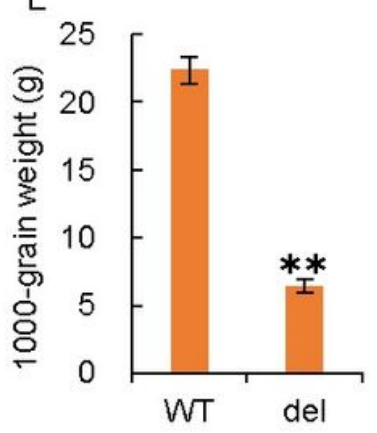

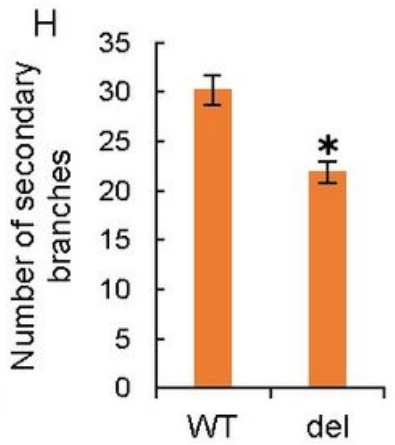

$\mathrm{M}$

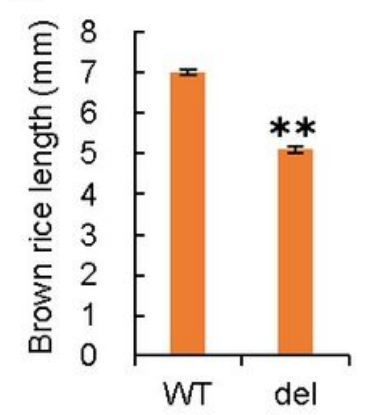

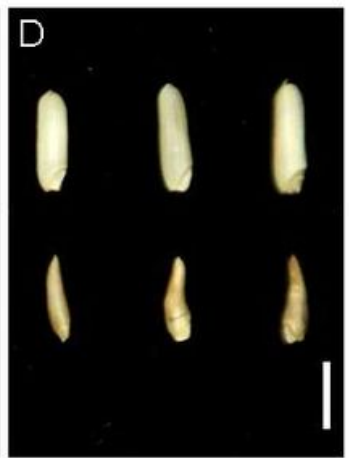

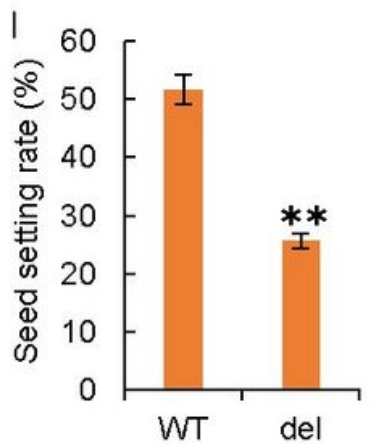

$\mathrm{N}$

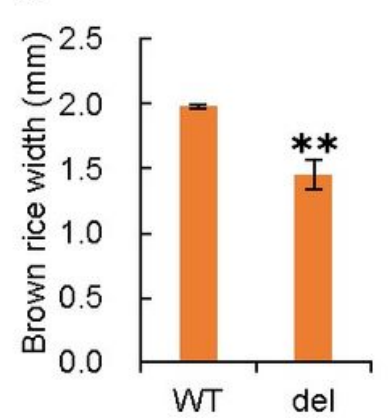

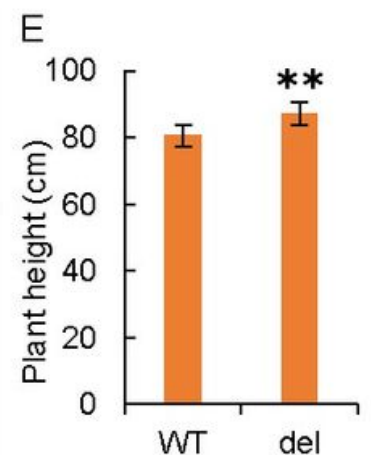
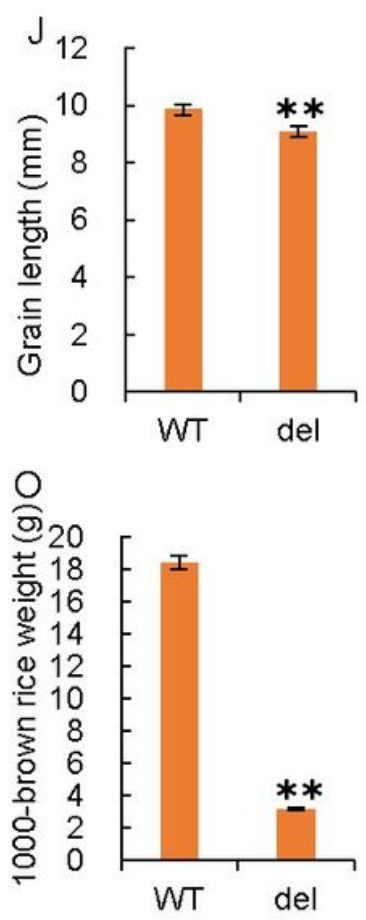

Figure 2

\section{Figure 2}

Investigation of yield-related agronomic traits in the wild type and de/ mutant. A, Plant type of the wild type (left) and de/mutant (right). B, Main panicle of the wild type (left) and de/ mutant (right). C, Mature grains of the wild type (upper), slightly degenerated del mutant (middle) and rod-like del mutant (lower). 
D, Brown rice of the wild type (upper) and slightly degenerated del mutant (lower). E-I, Plant height (E), panicle length $(F)$, number of primary branches $(G)$, number of secondary branches $(H)$, and seed setting rate $(\mathrm{I})$. J-L, length $(\mathrm{J})$, width $(\mathrm{K})$, and 1000-grain weight $(\mathrm{L})$ of mature grains in the wild type and del mutant. M-O, length $(\mathrm{M})$, width $(\mathrm{N})$, and 1000-grain weight $(0)$ of brown rice in the wild type and del mutant. $P$ value is determined using a t-test compared with 1B. ** represents $P \leq 0.01$. Error bars indicate standard deviation. $B a r=5 \mathrm{~cm}$ in $A$ and $B, 4 \mathrm{~mm}$ in $C$ and $D$.
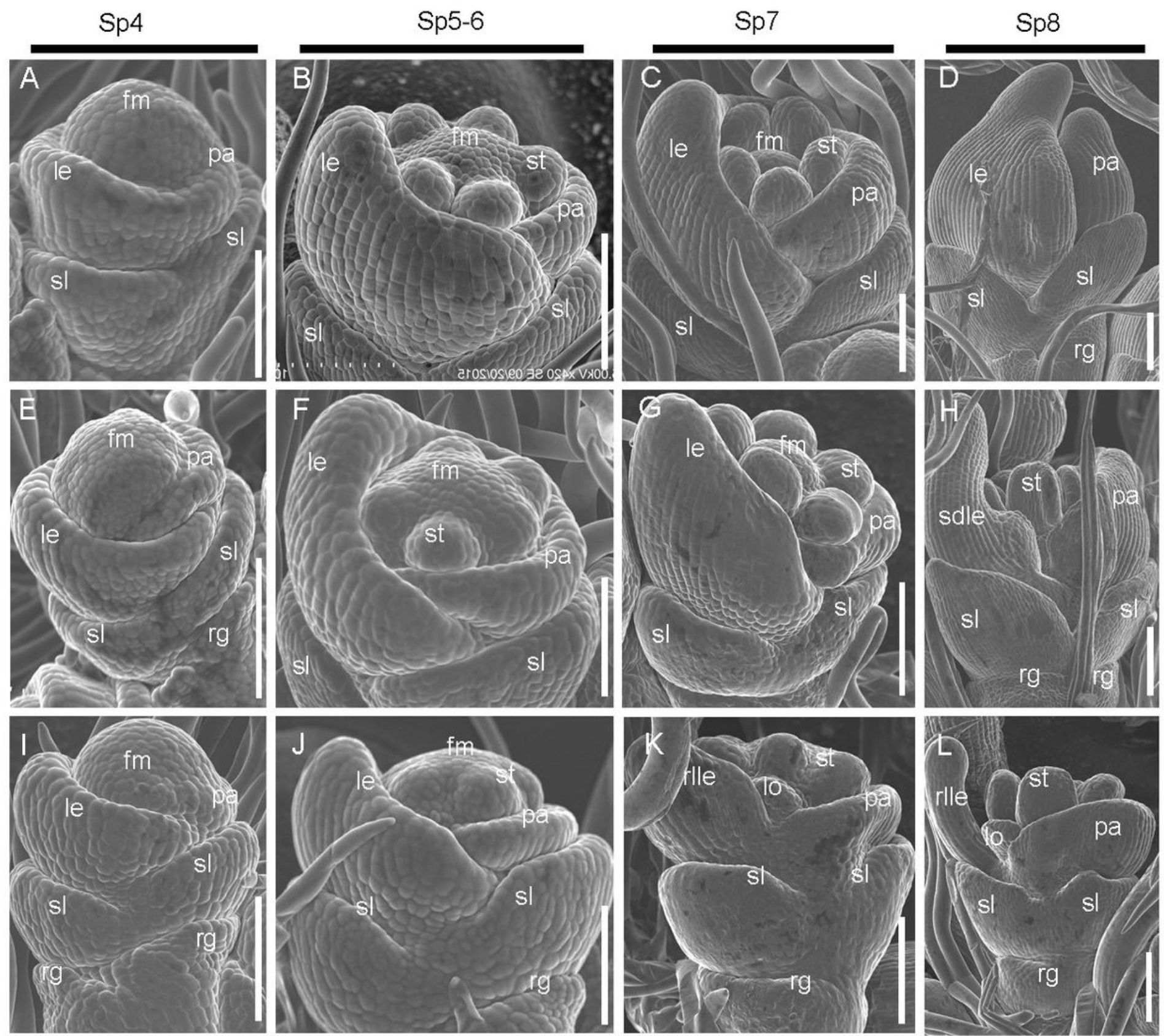

\section{Figure 3}

\section{Figure 3}


Spikelet phenotypes of the wild type and the de/ mutant. A-D, Spikelet of the wild type at Sp4-8, The del mutant spikelet at five developmental stages: E and I, Sp4; F and J, Sp5-Sp6; G and K, Sp7; H and L, Sp8. le, lemma; pa, palea; fm, floral meristem; st, stamen; rg, rudimentary glume; sl, sterile lemma; lo, lodicule; sdle: slightly degenerated lemma organ; rlle: rod-like lemma organ. Bars $=1 \mathrm{~mm}$.

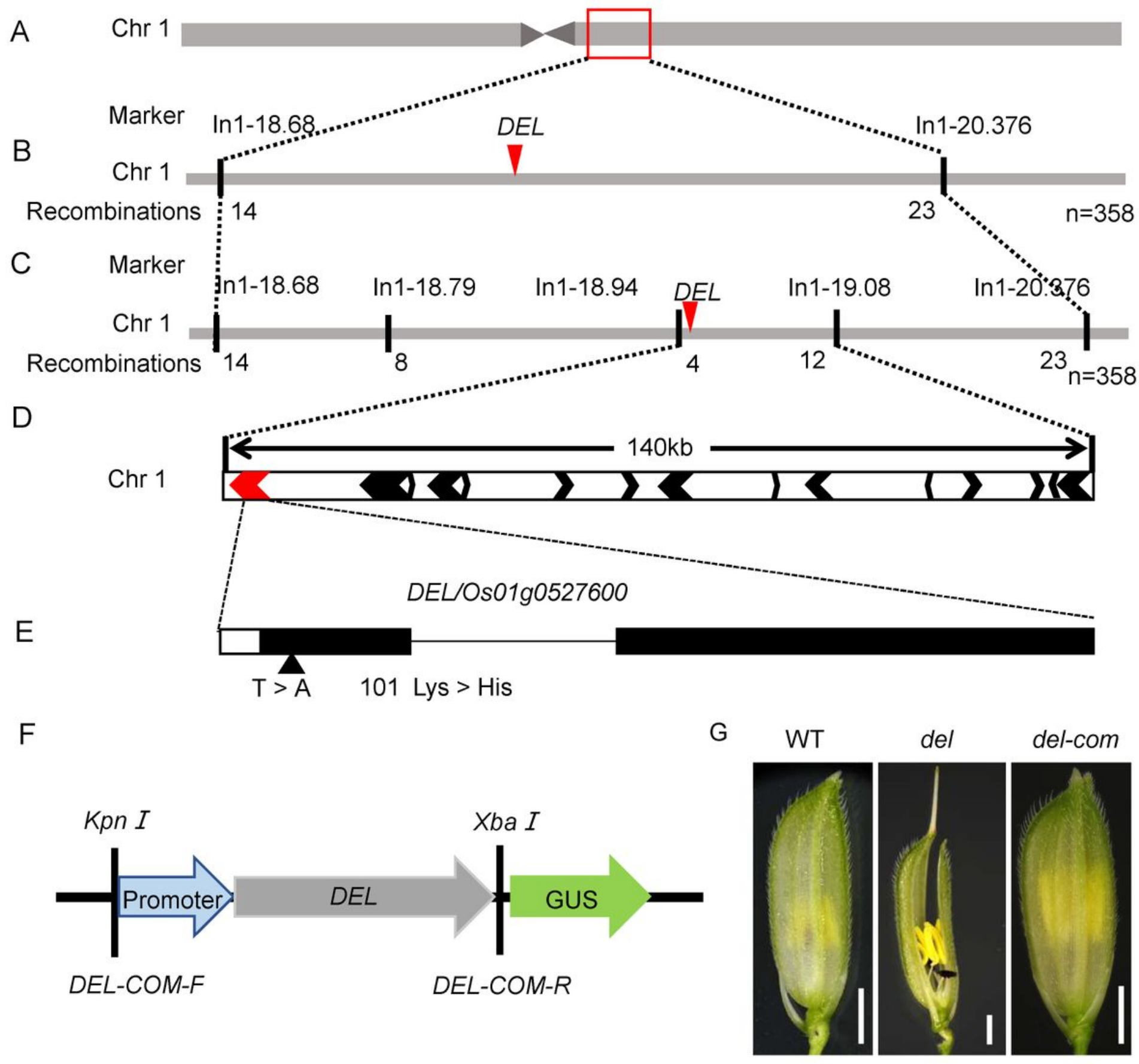

Figure 4

Figure 4

Localization of $D E L$ and candidate gene analysis. A and $\mathrm{B}$, Primary mapping of $D E L$ on chromosome 1 based on 358 individuals. C, DEL was fine-mapped to an interval of $140 \mathrm{~kb}$ using 358 individuals. D, Fifteen genes were annotated in the $140 \mathrm{~kb}$ region. $\mathrm{E}, \mathrm{A}$ single-nucleotide substitution from $\mathrm{T}$ to $\mathrm{A}$ was 
detected in 0s01g0527600.Mutation site of Os01g0527600 was between the de/mutant and the wild type 'Xinong 1B'. F, Schematic structure of the complementation vector pCAMBIA1301-DEL-GFP. G, The spikelets of WT, del, and the complementary transgenic line. Bars $=2000 \mu \mathrm{m}$.

A

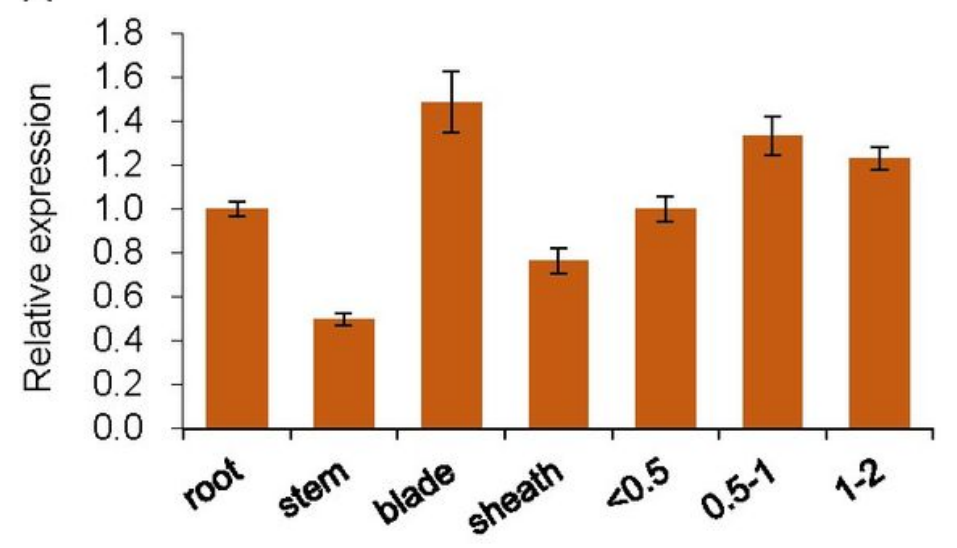

Sp4

Sp5-Sp6
B

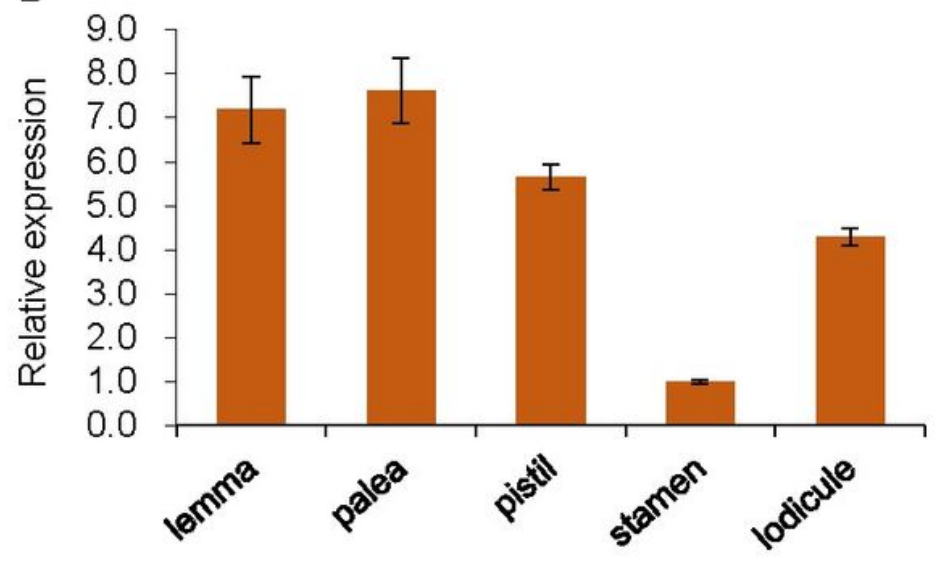

glume
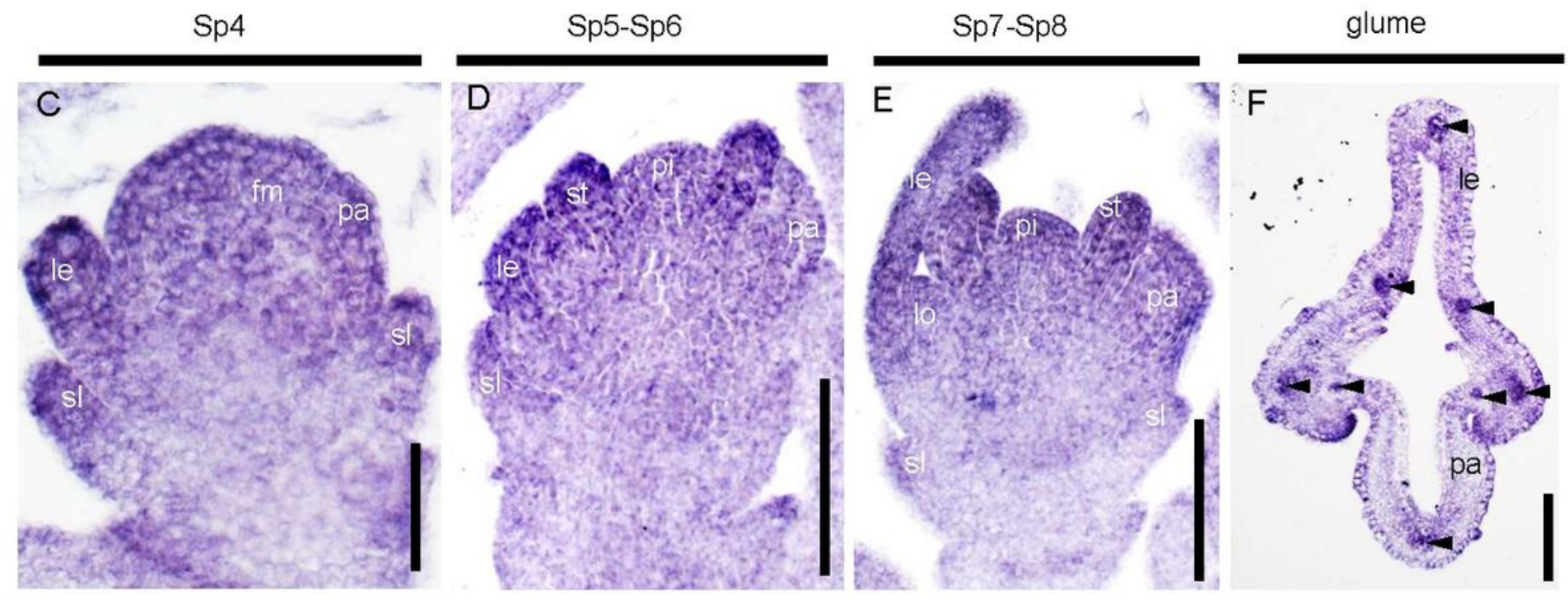

Figure 5

Figure 5

Spatiotemporal expression pattern of the $D E L$ gene.

A and B, RT-qPCR of DEL. ACTIN was used as a control. Young panicles $<0.5 \mathrm{~cm}, 0.5-1.0 \mathrm{~cm}, 1.0-2.0$ $\mathrm{cm}$, vegetative organ, and floral organ of the wild type were used. Data are Mean $\pm S D(n=3) . C-F$, Expression pattern of $D E L$ in spikelets of the wild type. In situ hybridization in the spikelets of the wild type during stages Sp3 (C), Sp4 (D), Sp5-7 (E) and Sp8 (F). fm, floral meristem; le, lemma; pa, palea; st, stamen; pi, pistil; rg, rudimentary glume; sl, sterile lemma. The black arrows indicate the vascular bundles. Bars $=100$ um. 

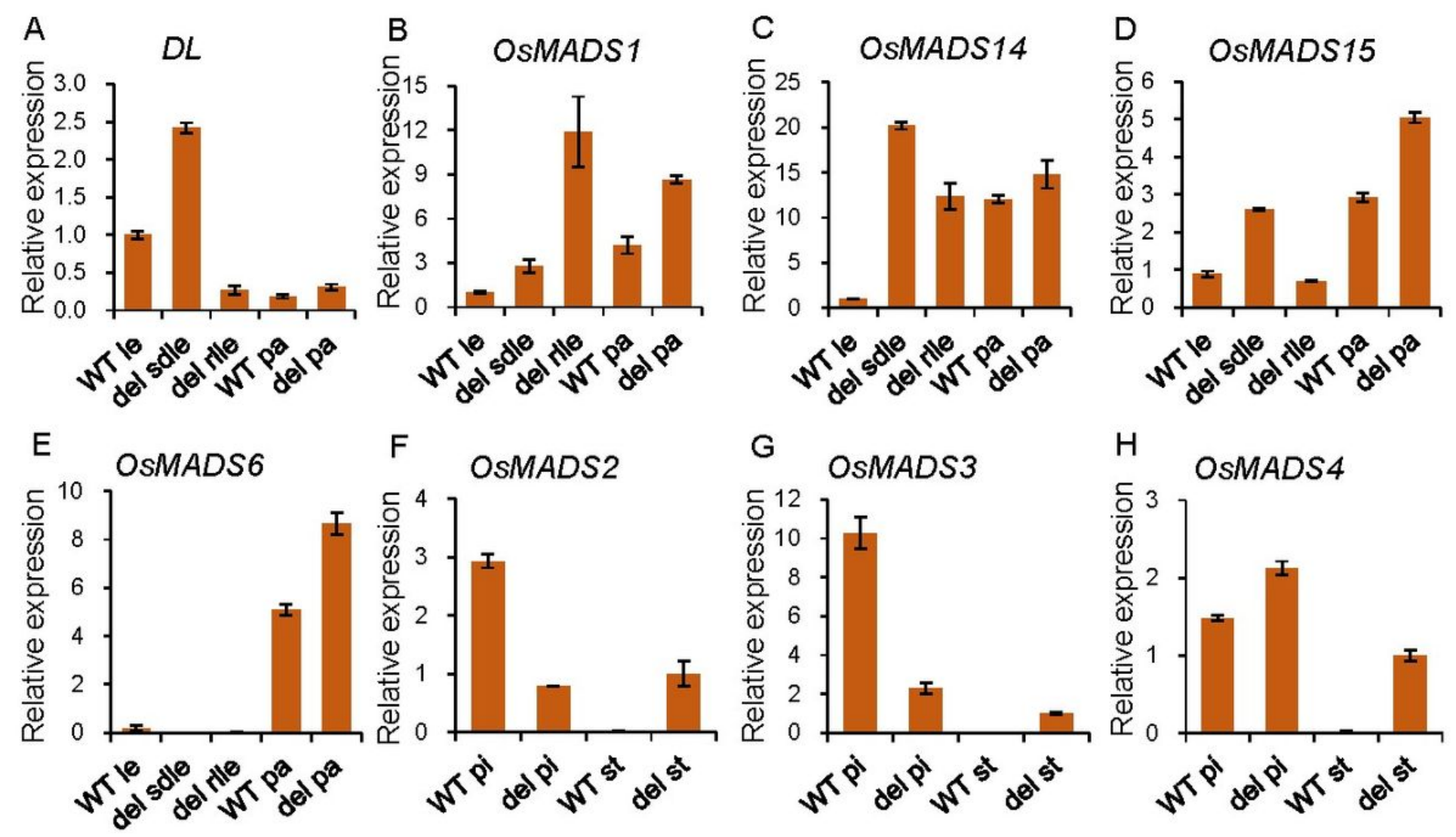

Figure 6

Figure 6

Relative expression levels of floral organ identity genes in floral organs of the wild type and de/ mutant. le, lemma; pa, palea; sdle: slightly degenerated lemma organ; rlle: rod-like lemma organ; st, stamen; pi, pistil.

\section{Supplementary Files}

This is a list of supplementary files associated with this preprint. Click to download.

- Supplementaltable.docx 